\title{
Corrigendum: Motley Crew: Overview of the Currently Available Phage Diversity
}

\author{
Nikita Zrelovs, Andris Dislers and Andris Kazaks* \\ Latvian Biomedical Research and Study Centre, Riga, Latvia
}

Keywords: complete genome, phage diversity, bioinformatics, genome overview, bacteriophage

\section{A Corrigendum on}

Motley Crew: Overview of the Currently Available Phage Diversity

by Zrelovs, N., Dislers, A., and Kazaks, A. (2020). Front. Microbiol. 11:579452. doi: $10.3389 /$ fmicb.2020.579452

In the original article, there were some errors.

Several corrections have been made in the "Situation as of today" section,

Second paragraph, second sentence:

Currently, ssDNA bacteriophages (families Microviridae, Inoviridae, Plectroviridae, Finnlakeviridae) are represented with 92 putative species complete genomes $(\sim 1.12 \%$ of the total genome count), dsDNA phages (Ackermannviridae, Autographiviridae, Chaseviridae,

OPEN ACCESS

Edited and reviewed by:

Robert Czajkowski,

University of Gdansk, Poland

*Correspondence:

Andris Kazaks

andris@biomed.lu.Iv

Specialty section:

This article was submitted to

Virology,

a section of the journal

Frontiers in Microbiology

Received: 06 November 2020 Accepted: 18 November 2020 Published: 06 January 2021

Citation:

Zrelovs N, Dislers A and Kazaks A (2021) Corrigendum: Motley Crew: Overview of the Currently Available

Phage Diversity.

Front. Microbiol. 11:626744. doi: 10.3389/fmicb.2020.626744
Demerecviridae, Drexlerviridae, Herelleviridae, Myoviridae, Podoviridae, Siphoviridae, Sphaerolipoviridae, Corticoviridae, Tectiviridae, Plasmaviridae) - 7736 genomes ( 93.83\%), dsRNA phages (Cystoviridae) - 7 genomes $(\sim 0.08 \%)$, and ssRNA phages (Leviviridae) -23 genomes $(\sim 0.28 \%)$, while the putative phage species unclassified at the family level are represented by 387 genomes corresponding to approximately $4.69 \%$ of the total genome number (Table 1).

Fifth paragraph, second sentence:

Myo- (long contractile tail) and podo- (short non-contractile tail) phages, on the other hand, are currently found in Myoviridae and Podoviridae, and also in Ackermannviridae, Herelleviridae, Chaseviridae (myophages), and Autographiviridae (podophages) phage families.

Several corrections have been made in the "Discussion" section:

First paragraph, fifth sentence:

Genome physical termini predictions (either by prediction tools or manual inspection of reads mapping onto the putative genome) require a large amount of individual phage reads and may present ambiguous results otherwise (Garneau et al., 2017).

First paragraph, eighth and ninth sentences:

Many of the submissions do not have any manuscript linked to it where the methodology would be stated in-detail, and the submission-associated metadata (that are sometimes very scarce) along with the functional annotation are not always enough to evaluate the plausibility of the "complete genome." This is raising additional concern for metagenomics acquired 
phage "complete genomes," evaluation of which should be handled with particular care, possibly including a brief evidence statement on why the submission authors are confident about the "completeness" of the entry in the sequence metadata (e.g., the "circularity" of the assembly).

Fourth paragraph, second sentence:

Taking the rate of complete phage genome submissions to public sequence repositories into account and addressing concerns about the future usability of entries in such repositories, we, sadly, have to stress the importance of taking the submission process seriously.

Fifth paragraph, first sentence:

First of all, there have been examples of typing errors in the metadata (e.g., "Eschericha" or "Panteoa" instead of the

\section{REFERENCES}

Garneau, J. R., Depardieu, F., Fortier, L. C., Bikard, D., and Monot, M. (2017). PhageTerm: A tool for fast and accurate determination of phage termini and packaging mechanism using next-generation sequencing data. Sci. Rep. 7, 1-10. doi: 10.1038/s41598-017-0 7910-5 correct Escherichia and Pantoea, "Vibro" instead of Vibrio, and Mycobacterium misspelled in multiple ways).

Sixth paragraph, first sentence:

Secondly, if the sequence of a phage (bacterial virus) is being submitted, submission authors should try to avoid the ambiguous usage of the sequence-related metadata qualifiers (e.g., "/host =" qualifier used for organisms other than bacteria); bacteriophages, being viruses of bacteria, infect and replicate within bacteria, which serve as a natural host.

Table 1, column "Family":

Replace "Drexlerviridae" for "Drexleviridae". The corrected Table 1 appears below.

The authors apologize for all the aforementioned typographic errors and state that they did not change the scientific conclusions of the article in any way.

Copyright (c) 2021 Zrelovs, Dislers and Kazaks. This is an open-access article distributed under the terms of the Creative Commons Attribution License (CC BY). The use, distribution or reproduction in other forums is permitted, provided the original author(s) and the copyright owner(s) are credited and that the original publication in this journal is cited, in accordance with accepted academic practice. No use, distribution or reproduction is permitted which does not comply with these terms. 
TABLE 1 | Overview of completely sequenced phage genomes.

\begin{tabular}{|c|c|c|c|c|c|c|c|}
\hline Family & $\begin{array}{l}\text { Complete } \\
\text { Putative Phage } \\
\text { Species Genome } \\
\text { Count }\end{array}$ & $\begin{array}{l}\text { Percent of the } \\
\text { Total Complete } \\
\text { Phage Genomes }\end{array}$ & Base pairs & $\begin{array}{l}\text { Percent of the } \\
\text { total base pairs }\end{array}$ & $\begin{array}{l}\text { Mean Genome } \\
\text { Length } \pm \text { SD (bp) }\end{array}$ & $\begin{array}{l}\text { Phage with the shortest annotated } \\
\text { genome/Accession/Genome Length (bp) }\end{array}$ & $\begin{array}{l}\text { Phage With the longest annotated } \\
\text { genome/Accession/Genome Length (bp) }\end{array}$ \\
\hline Siphoviridae & 4460 & $54.09 \%$ & 230398476 & $40.43 \%$ & $51659 \pm 23045$ & $\begin{array}{l}\text { Rhodococcus phage } \\
\text { RRH1/NC_016651.1/14270 bp * }\end{array}$ & $\begin{array}{l}\text { Caulobacter phage } \\
\text { CcrBL9/NC_048047.1/322272 bp }\end{array}$ \\
\hline Myoviridae & 1608 & $19.50 \%$ & 214998333 & $37.72 \%$ & $133705 \pm 80193$ & $\begin{array}{l}\text { Klebsiella phage } \\
\text { ST101-KPC2phi6.2/MK416016.1/11454 bp }\end{array}$ & $\begin{array}{l}\text { Prevotella phage Lak-B8/MK250027.1/551627 } \\
\text { bp }\end{array}$ \\
\hline Podoviridae & 571 & $6.93 \%$ & 28277096 & $4.96 \%$ & $49522 \pm 20777$ & $\begin{array}{l}\text { Pectobacterium phage } \\
\text { DU_PP_III/MF979562.1/11504 bp }\end{array}$ & $\begin{array}{l}\text { Cellulophaga phage } \\
\text { phi4:1_13/KT962245.1/145865 bp }\end{array}$ \\
\hline Autographiviridae & 481 & $5.83 \%$ & 20051636 & $3.52 \%$ & $41687 \pm 2468$ & $\begin{array}{l}\text { Klebsiella phage } \\
\text { PBKP05/MH885472.1/30240 bp }\end{array}$ & $\begin{array}{l}\text { Klebsiella virus 2019KP1/MT360680.1/48372 } \\
\text { bp }\end{array}$ \\
\hline Unknown family & 387 & $4.69 \%$ & 7559548 & $1.33 \%$ & $19534 \pm 23018$ & Leuconostoc phage L5/L06183.1/2435 bp & $\begin{array}{l}\text { Synechococcus phage } \\
\text { S-SCSM1/MK867354.1/228827 bp }\end{array}$ \\
\hline Herelleviridae & 219 & $2.66 \%$ & 32245234 & $5.66 \%$ & $147239 \pm 13265$ & $\begin{array}{l}\text { Bacillus phage Maceta/MH538296.1/45023 } \\
\text { bp }\end{array}$ & $\begin{array}{l}\text { Bacillus phage } \\
\text { AvesoBmore/NC_028887.1/167431 bp }\end{array}$ \\
\hline Drexlerviridae & 166 & $2.01 \%$ & 8034195 & $1.41 \%$ & $48399 \pm 4451$ & $\begin{array}{l}\text { Escherichia phage } \\
\text { IMM-001/MF630922.1/32486 bp * }\end{array}$ & $\begin{array}{l}\text { Klebsiella phage } \\
\text { vB_KpnS_Domnhall/MN013075.1/54438 bp }\end{array}$ \\
\hline Demerecviridae & 120 & $1.46 \%$ & 13476523 & $2.36 \%$ & $112304 \pm 13553$ & $\begin{array}{l}\text { Salmonella phage } \\
\text { GE_vB_N8/MG969413.1/51134 bp * }\end{array}$ & $\begin{array}{l}\text { Salmonella phage } \\
\text { GE_vB_N5/MG969412.1/148669 bp }\end{array}$ \\
\hline Ackermannviridae & 86 & $1.04 \%$ & 13446889 & $2.36 \%$ & $156359 \pm 4692$ & $\begin{array}{l}\text { Acinetobacter phage SH-Ab } \\
\text { 15599/MH517022.1/143204 bp }\end{array}$ & $\begin{array}{l}\text { Ralstonia phage RSP15/NC_030948.1/167619 } \\
\text { bp }\end{array}$ \\
\hline Inoviridae & 57 & $0.69 \%$ & 408238 & $0.07 \%$ & $7162 \pm 1205$ & $\begin{array}{l}\text { Uncultured phage } \\
\text { WW-nAnB/NC_026582.1/4817 bp }\end{array}$ & Vibrio phage CTX/NC_015209.1/10638 bp \\
\hline Microviridae & 29 & $0.35 \%$ & 153147 & $0.03 \%$ & $5281 \pm 652$ & $\begin{array}{l}\text { Ruegeria phage } \\
\text { VB_RpoMi-V15/MH015251.1/4248 bp }\end{array}$ & $\begin{array}{l}\text { Cellulophaga phage } \\
\text { phi12a:1/NC_021805.1/6478 bp }\end{array}$ \\
\hline Leviviridae & 23 & $0.28 \%$ & 88122 & $0.02 \%$ & $3831 \pm 365$ & $\begin{array}{l}\text { Enterobacteria phage BZ13 strain } \\
\text { T72/FJ483838.1/3393 bp }\end{array}$ & $\begin{array}{l}\text { Enterobacteria phage FI strain } \\
\text { BR1/FJ539134.1/4273 bp }\end{array}$ \\
\hline Tectiviridae & 11 & $0.13 \%$ & 169814 & $0.03 \%$ & $15032 \pm 2414$ & $\begin{array}{l}\text { Thermus phage phiKo/MH673671.2/11129 } \\
\text { bp }\end{array}$ & $\begin{array}{l}\text { Streptomyces phage } \\
\text { WheeHeim/MK305890.1/18266 bp }\end{array}$ \\
\hline Chaseviridae & 7 & $0.08 \%$ & 381319 & $0.07 \%$ & $54474 \pm 1190$ & $\begin{array}{l}\text { Escherichia phage } \\
\text { ST32/NC_047830.1/53092 bp }\end{array}$ & $\begin{array}{l}\text { Erwinia phage } \\
\text { vB_EamM-Y2/NC_019504.1/56621 bp }\end{array}$ \\
\hline Cystoviridae & 7 & $0.08 \%$ & 94655 & $0.02 \%$ & $13522 \pm 715$ & $\begin{array}{l}\text { Pseudomonas phage phi2954/L: NC_012091; } \\
\text { M: NC_012092; S: NC_012093/12685 bp }\end{array}$ & $\begin{array}{l}\text { Pseudomonas phage phi8/L: NC_003299; M: } \\
\text { NC_003300; S: NC_003301/14984 bp }\end{array}$ \\
\hline Plectroviridae & 5 & $0.06 \%$ & 35227 & $0.01 \%$ & $7045 \pm 1523$ & $\begin{array}{l}\text { Acholeplasma phage } \\
\text { MV-L1/NC_001341.1/4491 bp }\end{array}$ & $\begin{array}{l}\text { Spiroplasma phage } \\
\text { 1-R8A2B/NC_001365.1/8273 bp }\end{array}$ \\
\hline Corticoviridae & 4 & $0.05 \%$ & 40085 & $0.01 \%$ & $10021 \pm 831$ & $\begin{array}{l}\text { Marinomonas phage YY/MH105080.1/8828 } \\
\text { bp }\end{array}$ & Vibrio phage fNo16/MH730557.1/10594 bp \\
\hline $\begin{array}{l}\text { Sphaerolipoviridae (only } \\
\text { Bacterial viruses) }\end{array}$ & 2 & $0.02 \%$ & 36640 & $0.01 \%$ & $18320 \pm 1815$ & $\begin{array}{l}\text { Thermus phage P23-77/NC_013197.1/17036 } \\
\text { bp }\end{array}$ & $\begin{array}{l}\text { Thermus thermophilus phage } \\
\text { IN93/NC_004462.2/19604 bp }\end{array}$ \\
\hline Finnlakeviridae & 1 & $0.01 \%$ & 9174 & $0.00 \%$ & $9174 \pm 0$ & Flavobacterium phage FLiP/NC_047837.1/9174 bp & \\
\hline Plasmaviridae & 1 & $0.01 \%$ & 11965 & $0.00 \%$ & $11965 \pm 0$ & Acholeplasma phage L2/NC_001447.1/11965 bp & \\
\hline Overall & 8245 & $100.00 \%$ & 569916316 & $100.00 \%$ & $69163 \pm 55772$ & Leuconostoc phage L5/L06183.1/2435 bp & $\begin{array}{l}\text { Prevotella phage Lak-B8/MK250027.1/551627 } \\
\text { bp }\end{array}$ \\
\hline
\end{tabular}

Asterisk near entry in the column representing the shortest genome of a phage of a given family indicates the shortest plausible entry and ignores ambiguous entries labeled as "complete genome". 